\title{
Radiation and anticancer drugs can facilitate mitochondrial bypass by CD95/Fas via c-FLIP downregulation
}

\author{
I Verbrugge ${ }^{1}$, C Maas ${ }^{1}$, M Heijkoop ${ }^{1}$, M Verheij ${ }^{2}$ and J Borst ${ }^{\star, 1}$
}

In many tumor cell types, ionizing radiation or DNA-damaging anticancer drugs enhance sensitivity to death receptor-mediated apoptosis, which is of clinical interest. APO010, a form of CD95/Fas ligand is currently in a phase I trial in patients with solid tumors. To analyze the potential of combined modality treatment with APO010, we used p53-mutant Jurkat T leukemic cells, in which the mitochondrial pathway was blocked by Bcl-2 overexpression. These cells were strongly sensitized to APO010 by pretreatment with ionizing - or UV radiation, etoposide, histone deacetylase - or proteasome inhibitors. These stimuli alone did not induce apoptosis in J16-Bcl-2 cells. Sensitization could not be explained by the overruling of mitochondrial resistance imposed by $\mathrm{Bcl}-2$, upregulation of CD95 membrane levels or modulation of inhibitor of apoptosis proteins. Rather, the stimuli commonly downregulated $C$-FLIP $P_{L / S}$ protein levels, which was causally related to the sensitization: deliberate $c$-FLIP $P_{L / S}$ downregulation by RNA interference largely overruled the capacity of the various stimuli to sensitize Jurkat-Bcl-2 cells to apoptotic execution by APO010. In p53-mutant, Bcl-2 overexpressing HCT-15 colon carcinoma cells, c-FLIP downregulation correlated with sensitization to APO010 for some, but not all stimuli. We conclude that C-FLIP downregulation represents a mechanism by which diverse anticancer regimens can facilitate tumor cell execution by CD95/Fas through the direct pathway of caspase activation.

Cell Death and Differentiation (2010) 17, 551-561; doi:10.1038/cdd.2009.141; published online 2 October 2009

Inhibition of apoptotic pathways critically contributes to cancer development. Consequently, tumor cells are often 'addicted' to overexpression of anti-apoptotic proteins, such as Bcl-2, for their survival. Targeting apoptosis regulators in cancer treatment is therefore interesting, as overcoming blocks in apoptosis may render cancer cells more susceptible to death than normal cells. ${ }^{1}$ Ionizing radiation (IR) and DNA-damaging chemotherapeutics are effective in cancer treatment, because they hamper clonogenicity of tumor cells in a variety of ways. The DNA damage that they induce triggers an often p53-dependent cell cycle arrest allowing for DNA repair. Failing this, irreversible cell cycle arrest, death due to mitotic catastrophe or apoptotic cell death may ensue. ${ }^{2}$ Apoptosis induction by DNA-damaging anticancer regimens is blocked by $\mathrm{Bcl}-2$ overexpression, indicating that apoptosis signalling proceeds through the intrinsic, mitochondrial pathway. ${ }^{3}$ In this pathway, pro-apoptotic members of the Bcl-2 family induce mitochondrial permeabilization, and the subsequent release of cytochrome $c($ Cyt $c)$ and other mediators allows for Caspase- 9 and effector caspase activation. ${ }^{4}$

In hematopoietic tumors, the apoptotic response to IR and DNA-damaging drugs is generally evident, and apoptosis resistance can contribute to treatment resistance. ${ }^{5}$ Solid tumors are less apoptosis prone when treated with conventional regimens. Therefore, the death receptor ligand TRAIL was of immediate clinical interest when it effectively induced apoptosis in many long-term established solid tumor cell lines. TRAIL proved non-toxic to normal tissue in animal models and moved rapidly toward the clinical testing phase. ${ }^{6}$ Currently, TRAIL receptor agonists are in phases I and II clinical trials, either as single modality treatment or in combination with conventional or novel anticancer drugs. ${ }^{7}$

Combined modality treatment with death receptor agonists and DNA-damaging anticancer regimens was expected to be more effective than single-agent treatment, given the distinct cell death pathways they engage. TRAIL-R1/2 and CD95 death receptors induce apoptosis by directly recruiting and activating caspases. ${ }^{8}$ Upon ligand binding, they recruit FADD, inducer caspase-8 and/or caspase-10 and c-FLIP molecules. $^{8,9}$ In the death-inducing signalling complex (DISC) thus formed at the cytoplasmic receptor tail, caspase-8/10 are activated. Depending on their expression level, c-FLIP molecules can facilitate or inhibit this activation. ${ }^{10}$ Upon selfprocessing by proteolysis, caspase-8/10 are released into the cytosol, in which they find effector caspases as their targets. In this way, death receptors can in principle execute apoptosis

\footnotetext{
${ }^{1}$ Division of Immunology, The Netherlands Cancer Institute/Antoni van Leeuwenhoek Hospital, Amsterdam, The Netherlands and ${ }^{2}$ Division of Radiotherapy, The Netherlands Cancer Institute/Antoni van Leeuwenhoek Hospital, Amsterdam, The Netherlands

${ }^{*}$ Corresponding author: J Borst, Division of Immunology, The Netherlands Cancer Institute, Plesmanlaan 121, Amsterdam 1066 CX, The Netherlands.

Tel: + 3120 5122056; Fax: + 3120 5122057; E-mail: j.borst@nki.nl

Keywords: CD95/Fas; apoptosis; c-FLIP; mitochondria; type II; Bcl-2

Abbreviations: c-FLIP, cellular FLICE-like inhibitory protein; DISC, death-inducing signalling complex; FADD, Fas-associated protein with death domain; HDAC, histone deacetylase; IAP, inhibitor of apoptosis protein; JNK, Jun N-terminal kinase; L, ligand; mAb, monoclonal antibody; R, receptor; RNAi, RNA interference; TNF, tumor necrosis factor; TRAIL, TNF-related apoptosis-inducing ligand; TSA, trichostatin A; UV, ultraviolet; VPA, Valproic acid

Received 27.2.09; revised 31.8.09; accepted 01.9.09; Edited by J Silke; published online 02.10.09
} 
in a mitochondrion-independent manner. However, by cleaving and activating BH3-only protein, Bid, caspase-8/10 also connect death receptors to the mitochondrial pathway. ${ }^{4}$

The efficacy of TRAIL receptors and CD95 to kill cells through the direct pathway for effector caspase activation depends on the cell type. In the so-called type II cells, the mitochondrial contribution is required for apoptotic execution, whereas in type I cells it is not, as ascertained by $\mathrm{Bcl}-2$ overexpression. This relates to the efficacy of caspase-8/10 activation in the DISC, which seems a cell-intrinsic phenomenon. ${ }^{11}$ The mitochondrial bypass by death receptors is of interest, as it potentially overrules intrinsic apoptosis resistance in tumor cells, as for example, imposed by $\mathrm{Bcl}-2$ overexpression.

Although TRAIL receptors and CD95/Fas induce apoptosis by highly similar mechanisms, ${ }^{8,9}$ CD95 agonists were not pursued for cancer therapy, because of severe hepatotoxicity upon systemic administration in mice. ${ }^{12}$ Recently however, a phase I clinical trial has been initiated at TopoTarget (ClinicalTrials.gov identifier: NCT00437736) with APO010 (MegaFas Ligand). This is a fusion protein of the extracellular domain of CD95L/FasL and the collagen domain of the serum protein ACRP30. The dimer of CD95L trimers that is thus formed can crosslink two adjacent CD95 trimers, rendering APO010 highly agonistic as compared with other available CD95 agonists. ${ }^{13}$ It is therefore envisioned that a combined modality treatment with low concentrations of this agent is effective and has acceptable toxicity, especially in settings of local therapy, such as radiotherapy or local perfusion with anticancer drugs. We have recently shown that in p53-mutant Jurkat cells engineered to overexpress Bcl-2 (J16-Bcl-2), as well as in solid tumor types, APO010 and IR had a combined effect on cell death induction, both in short-term apoptosis assays and in clonogenic survival assays. ${ }^{14}$

In this study we show that pre-treatment with not only IR, but also with UV radiation and various anticancer drugs sensitizes J16-Bcl-2 cells to APO010-mediated apoptosis, without breaking mitochondrial resistance imposed by $\mathrm{Bcl}-2$ or upregulating CD95 membrane levels. The underlying mechanism concerns downregulation of c-FLIP long $(L)$ and short (S) isoforms, which enables apoptotic execution by CD95 through the direct pathway for caspase activation. As Jurkat cells are classified as type II cells, ${ }^{15}$ this implies a conversion from type II to type I signalling by CD95.

\section{Results}

Combining APO010 with chemotherapy overcomes apoptosis resistance imposed by $\mathrm{Bcl}-2$. The wild-type Jurkat clone $\mathrm{J} 16$ readily undergoes apoptosis upon single treatment with the death ligands, CD95/FasL or TRAIL, or with the DNA-damaging anticancer regimens, IR or etoposide. $^{16}$ The response of $\mathrm{J} 16$ cells to each of these stimuli was strongly impaired upon Bcl-2 overexpression. ${ }^{14,16}$ J16-Bcl-2 cells therefore provided an excellent cellular system to examine whether combined treatment with APO010 and anticancer regimens might overrule resistance imposed by Bcl-2. APO010 and IR were previously shown to have a combined effect on the apoptotic response of J16-Bcl-2 cells. ${ }^{14}$ To analyze whether this also held true for DNAdamaging drugs, we examined the combined effects of APO010 and etoposide, which is a topoisomerase inhibitor that - similar to IR - induces double-strand DNA breaks. The cells were stimulated with different doses of APO010 in the presence or absence of etoposide and apoptosis was read out after $48 \mathrm{~h}$ by nuclear fragmentation. J16-Bcl-2 cells were fully resistant to treatment with $2 \mu \mathrm{g} / \mathrm{ml}$ etoposide alone (Figure 1a). As expected from the type II nature of Jurkat cells, ${ }^{15} \mathrm{~J} 16-\mathrm{Bcl}-2$ cells also showed low sensitivity to AP0010 alone. In agreement with our previous work ${ }^{16}$ and studies by others, ${ }^{17,18} \mathrm{Bcl}-2$ overexpression did not fully abrogate the apoptotic response of J16 cells to CD95 stimulation, as evident at high doses of APO010 (Figure 1a). Upon combined treatment with etoposide, however, J16-Bcl-2 cells were significantly more sensitive to APO010 and underwent apoptosis at very low doses (Figure 1a). Wild-type J16 cells were highly sensitive to treatment with $2 \mu \mathrm{g} / \mathrm{ml}$ etoposide alone, which precluded a read out for sensitization to APO010 (Supplementary Figure 1). To analyze whether etoposide pre-conditioned J16-Bcl-2 cells to more efficiently activate effector caspases upon APO010 stimulation, the cells were first incubated with etoposide and $15 \mathrm{~h}$ later stimulated with APO010. Whereas APO010 alone readily induced caspase- 3 activation in parental $\mathrm{J} 16$ cells, J16-Bcl-2 cells were relatively resistant. In etoposide-pretreated $\mathrm{J} 16-\mathrm{Bcl}-2$ cells, caspase-3 activation upon APO010 treatment was significantly enhanced, approximating the response observed in $\mathrm{J} 16$ cells by APO010 treatment alone (Figure 1b). Caspase-3 activation correlated with the cell death response as measured by nuclear fragmentation and propidium iodide (PI) uptake (Supplementary Figure 2).

As effector caspases are directly activated by caspase-8/10 in the mitochondrion-independent death receptor pathway, we analyzed whether pre-treatment with etoposide improved the capacity of APO010 to activate caspase-8. In etoposidetreated $\mathrm{J} 16-\mathrm{Bcl}-2$ cells, caspase- 8 activation - as read out by appearance of the p41/p43 and p18 cleavage products - was first observed at $2 \mathrm{~h}$ after APO010 stimulation (Figure 1c). Caspase-3 activity was also apparent by proteolytic processing of its substrate PARP. At each subsequent time point, caspase-8 activation and PARP processing were more pronounced in cells incubated with etoposide than in untreated cells.

We conclude that etoposide treatment conditioned J16-Bcl-2 cells to more efficiently activate inducer and effector caspases upon APO010 stimulation.

Mitochondrial resistance imposed by $\mathrm{Bcl}-2$ is not broken upon combined treatment. It was possible that in etoposide-treated cells, APO010 evoked effector caspase activation by overruling the mitochondrial resistance imposed by $\mathrm{Bcl}-2$. Therefore, we monitored Cyt $c$ release in parental $\mathrm{J} 16$ cells and in $\mathrm{J} 16-\mathrm{Bcl}-2$ cells that were pretreated with etoposide or not. J16 cells showed Cyt $c$ release upon APO010 treatment but J16-Bcl-2 cells did not, throughout the 8-h time course, showing the effectiveness of $\mathrm{Bcl}-2$ in preventing mitochondrial permeabilization (Figure 2a). The primary data depicted in Figure $2 \mathrm{~b}$ illustrate this point. 

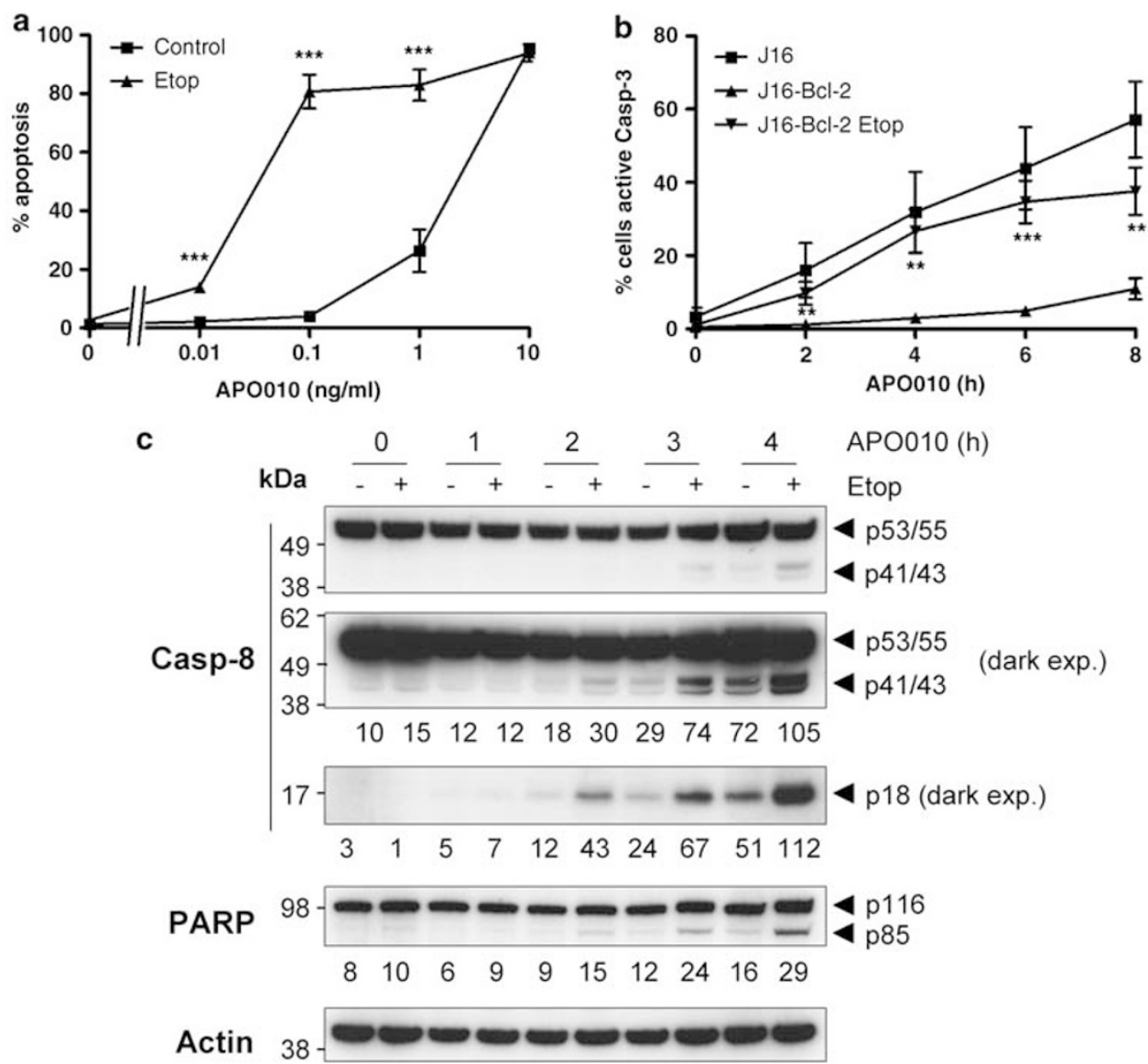

Figure 1 The combined effects of etoposide and APO010 on apoptosis induction in J16-Bcl-2 cells. (a) J16-Bcl-2 cells were stimulated with the indicated doses of APO010 in the absence (Control) or presence of $2 \mu \mathrm{g} / \mathrm{ml}$ etoposide (Etop). Apoptosis was read out by nuclear fragmentation after $48 \mathrm{~h}$. Results shown are means \pm S.D. of two independent experiments with duplicate samples. Statistically significant differences between values of Control and Etop samples are indicated as ${ }^{\star \star *} P<0.001$. (b) J16-Bcl-2 cells were left untreated (J16-Bcl-2) or incubated with $2 \mu \mathrm{g} / \mathrm{ml}$ etoposide (J16-Bcl-2 Etop). After $15 \mathrm{~h}$, the cells were stimulated with $0.4 \mathrm{ng} / \mathrm{ml}$ APO010. Empty vectortransduced J16 cells (J16) treated with APO010 alone served as a control. Active caspase-3 content was analyzed at the indicated time points using flow cytometry. Results shown are means \pm S.D. of three independent experiments. Statistically significant differences between values of J16-Bcl-2 and J16-Bcl-2 Etop samples are indicated as ${ }^{* \star} P<0.01$ and ${ }^{* *} P<0.001$. (c) J16-Bcl-2 cells were left untreated $(-)$ or stimulated with $2 \mu \mathrm{g} / \mathrm{ml}$ etoposide $(+)$. After $15 \mathrm{~h}(t=0)$, the cells were stimulated with $0.4 \mathrm{ng} / \mathrm{ml}$ APO010 and harvested at the indicated time points. After separation by SDS-PAGE, protein levels of the p53/55 caspase-8 proform and its p41/43 and p18 cleavage products and the PARP p 116 proform and its p85 cleavage product were determined using immunoblotting. Actin served as a loading control. The numbers indicate integrated density values of the caspase-8 p41/43, caspase-8 p18 and PARP p85 fragments, as quantified from autoradiograms and normalized to Actin signals in arbitrary units

Importantly, J16-Bcl-2 cells that were pretreated with etoposide showed no significant increase in Cyt $c$ release after APO010 stimulation, even after $8 \mathrm{~h}$ (Figure 2b). These were the same samples analyzed for caspase- 3 activation in Figure $1 \mathrm{~b}$, indicating that effector caspase activation in etoposide-treated $\mathrm{J} 16-\mathrm{Bcl}-2$ cells was not a consequence of breaking Bcl-2-mediated mitochondrial resistance. We conclude that after etoposide treatment, APO010 more efficiently activated effector caspases through the direct, mitochondrion-independent pathway.

Etoposide downregulates C-FLIP L/S $_{\text {S }}$ and improves caspase-8 activation in the DISC. As caspase-8 is activated in the DISC, we analyzed whether DISC formation was improved in etoposide-treated cells. After $15 \mathrm{~h}$ of incubation in the presence or absence of etoposide, J16-Bcl-2 cells were stimulated with crosslinked CD95L. The combined treatment effect in this setting was verified using enhanced PARP processing in corresponding total cell lysates (Figure 3a).

The DISCs isolated from etoposide pretreated cells showed enhanced caspase- 8 activation as compared to the DISCs from control cells, which was most apparent at the 4-h time point (Figure 3a). In the same samples, the DISCs from etoposide-treated cells showed a decrease in processed C-FLIP $\mathrm{P}_{\mathrm{L}}$ levels. Immunoblotting of total cellular lysates of control and etoposide-treated cells for c-FLIP levels showed that etoposide treatment reduced protein expression of the FLIP $_{L}$ and FLIPS isoforms (Figure $3 b$ ). This was a posttranslational effect, as c-FLIP mRNA levels were not reduced after etoposide treatment (Supplementary Figure 3).

These results suggest that downregulation of c-FLIP protein levels by etoposide treatment enhanced the ability of CD95L to activate caspase-8 in the DISC.

JNK inhibition reveals the relation between c-FLIP downregulation and sensitization to APO010. It was 


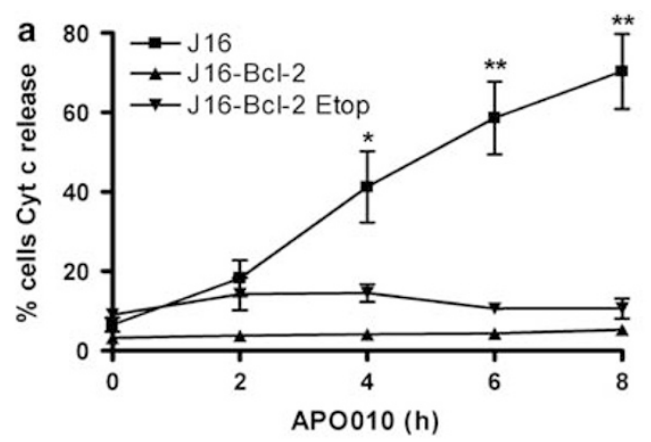

b J16 J16-Bcl-2
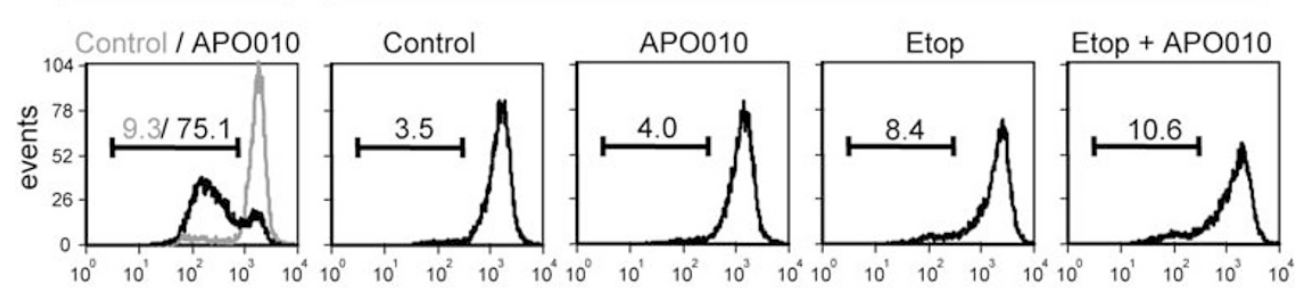

Cytochrome c (FI)

Figure 2 The combined treatment with etoposide and APO010 results in effector caspase activation in the absence of Cyt $c$ release. (a) Cyt $c$ release from the mitochondria was monitored in the same samples as analyzed for caspase- 3 activation in Figure 1b. The results shown are means \pm S.D. of three independent experiments. Statistically significant differences between the values of J16-Bcl-2 and J16-Bcl-2 Etop samples are indicated as ${ }^{*} P<0.05$ and ${ }^{* *} P<0.01$. (b) Flow cytometric plots showing Cyt $c$ content in $\mathrm{J} 16-\mathrm{Bcl}-2 \mathrm{cells}$ from one representative experiment at $8 \mathrm{~h}$ after the indicated treatments (Control equals mock incubation). Empty vector-transduced $\mathrm{J} 16$ cells (J16) served as a control for Cyt $c$ release. The numbers indicate percentage of cells with Cyt $c$ loss from mitochondria. Fl, fluorescence intensity

shown previously that activation of the Jun N-terminal kinase (JNK) pathway through activation of the E3 ubiquitin ligase Itch leads to c-FLIP downregulation. ${ }^{19}$ As etoposide is known to activate the JNK pathway, for example, ${ }^{20}$ we tested whether JNK inhibition - using the chemical inhibitor SP $600125^{21}$ or the cell permeable L-JNKi peptide ${ }^{22}$ -reversed etoposide-induced c-FLIP downregulation and APO010 sensitivity. Etoposide activated JNK in J16-Bcl-2 cells, as both JNK and its target protein c-Jun were phosphorylated upon treatment (Supplementary Figure 4). JNK activity induced by etoposide was strongly reduced by SP600125 and to a lesser extent by L-JNKi peptide, validating the compounds as JNK inhibitors. In the same experiment, etoposide treatment downregulated c-FLIP LS $_{\text {S }}$ protein levels. SP600125 and L-JNKi peptide restored c-FLIP protein levels to an extent that correlated with their inhibitory effect on JNK activity.

Next, we examined the effect of SP600125 on etoposidemediated sensitization of J16-Bcl-2 cells to CD95L. J16-Bcl-2 cells were pre-treated with etoposide or not, in the presence or absence of the JNK inhibitor. In total cellular lysates and in the CD95L DISC, C-FLIP $\mathrm{L}_{\mathrm{L}}$ was downregulated by etoposide treatment. However, co-treatment with SP600125 abrogated this effect, as observed at $t=0$ and at each successive time point after CD95L stimulation in total cell lysates and at 1 and $4 \mathrm{~h}$ in the DISC (Figure 4a). Concomitantly, SP600125 reversed etoposide-induced sensitization to CD95L, as the increment in caspase-8 activation and effector caspase activity (PARP cleavage) observed in etoposide-treated cells did not occur in cells that had been treated with etoposide and SP600125.
The effect of the JNK inhibitors on etoposide-mediated sensitization of J16-Bcl-2 cells to APO010-induced caspase-3 activation was validated using flow cytometry over an $8 \mathrm{~h}$ time course. Etoposide strongly sensitized J16-Bcl-2 cells to APO010-induced caspase-3 activation, which was efficiently reversed by SP600125 and also partially by L-JNKi peptide (Figure 4b). The ability of SP600125 and L-JNKi to reverse etoposide-mediated sensitization to APO010 correlated with their ability to inhibit c-Jun phosphorylation and to restore c-FLIP levels (Supplementary Figure 4).

These results indicate that in $\mathrm{J} 16-\mathrm{Bcl}-2$ cells, etoposidemediated activation of the JNK pathway is (partly) responsible for c-FLIP downregulation. Moreover, they suggest that c-FLIP downregulation by etoposide is causally related to the drug's ability to sensitize $\mathrm{J} 16-\mathrm{Bcl}-2$ cells to APO010induced apoptosis.

Downregulation of C-FLIP by RNA interference sensitizes $\mathbf{J 1 6 - B C l - 2}$ cells to APO010. To test whether c-FLIP downregulation was sufficient to sensitize J16-Bcl-2 cells to APO010-induced apoptosis, we stably downregulated c-FLIP levels in these cells by RNA interference (RNAi), using retroviral constructs. The different short interfering (si) RNA constructs tested downregulated c-FLIP isoforms to a variable degree. The FLIP1 construct strongly reduced the expression of $\mathrm{C}-\mathrm{FLIP}_{\mathrm{L}}$ and FLIP $_{\mathrm{S}}$, whereas for example, the FLIP4 construct had a clear, but more modest effect (Figure $5 \mathrm{a}$ ).

Upon treatment of FLIP1 and FLIP4 cell lines with APO010, caspase-3 activation was enhanced as compared with empty 
a

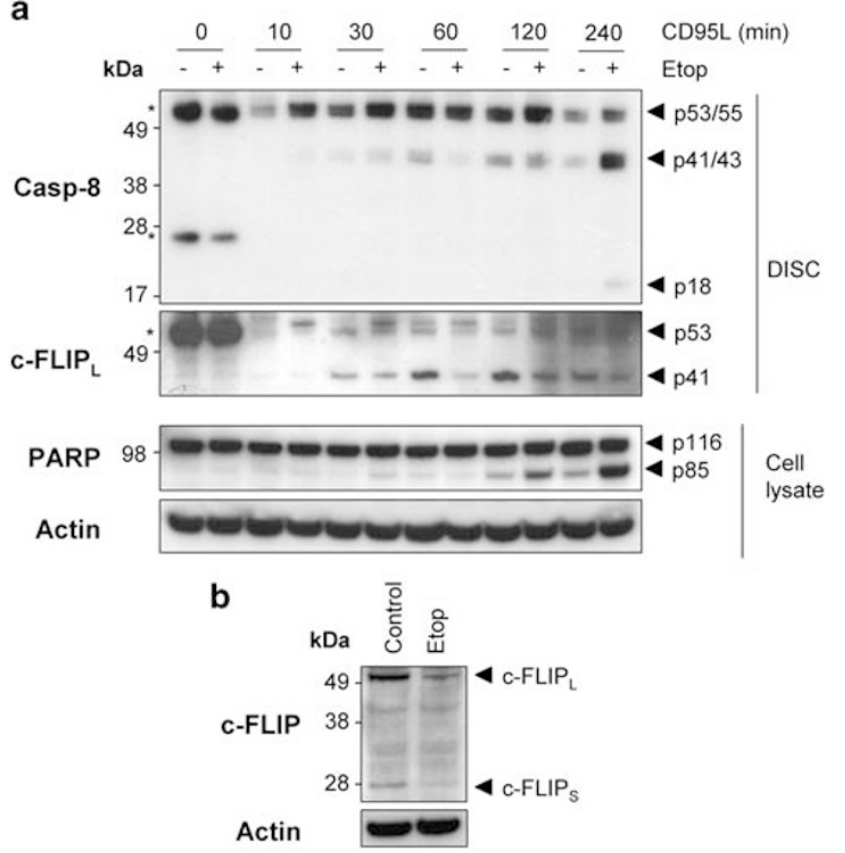

Figure 3 Etoposide promotes CD95L-induced caspase-8 activation in the DISC and reduces C-FLIP levels. (a) J16-Bcl-2 cells were incubated with $2 \mu \mathrm{g} / \mathrm{ml}$ etoposide $(+)$ or left untreated $(-)$. After $15 \mathrm{~h}$, the cells were stimulated with $20 \mathrm{ng} / \mathrm{ml}$ FLAG-CD95L pre-coupled to $100 \mathrm{ng} / \mathrm{ml}$ biotinylated anti-FLAG antibody for the indicated periods of time. CD95L-bound receptor complexes were isolated using streptavidin-conjugated sepharose beads. Caspase-8 and c-FLIP in the CD95L DISC were detected using immunoblotting. The caspase- 8 proforms (p53/55) and their cleavage products ( $p 41 / 43, p 18)$, as well as the c-FLIPL proform ( $p 53$ ) and its cleavage product ( $\mathrm{p} 41)$ are indicated. The combined effects in this setting were confirmed using immunoblotting, corresponding cell lysates for PARP processing (conversion of p116 to p85) as a measure for effector caspase activity. Actin served as a loading control. (b) Protein levels of c-FLIP $L / S$ in total lysates of J16-Bcl-2 cells were detected using immunoblotting at $15 \mathrm{~h}$ after incubation in the absence (Control) or presence of $2 \mu \mathrm{g} / \mathrm{ml}$ etoposide (Etop). Actin served as a loading control

vector-transduced cells to a degree that correlated with the extent of c-FLIP downregulation (Figure $5 b$ ). As tested in the FLIP1-transduced cells, caspase- 3 activation at $8 \mathrm{~h}$ was not accompanied with Cyt $c$ release, indicating that mitochondrial resistance was not broken (Figure $5 \mathrm{c}$ ).

We next analyzed to which extent etoposide treatment could (further) sensitize control, FLIP1- or FLIP4-transduced J16-Bcl-2 cells to APO010-induced apoptosis. After pretreatment with etoposide, cells were stimulated for $8 \mathrm{~h}$ with APO010. Control cells were efficiently sensitized to APO010 by etoposide in a dose-dependent manner. FLIP1-transduced cells were very sensitive to treatment with APO010 alone, and etoposide treatment incremented the response to a limited degree. FLIP4-transduced cells were sensitized to a lesser extent by c-FLIP siRNA, allowing us to quantify the added effect of etoposide pretreatment (Figure 5d). For this purpose, caspase-3 activation upon combined treatment was normalized to caspase- 3 activation by APO010 alone and expressed as fold sensitization. This analysis revealed that etoposidemediated sensitization to APO010 in FLIP4-transduced cells was significantly reduced as compared with control cells (Figure 5d).

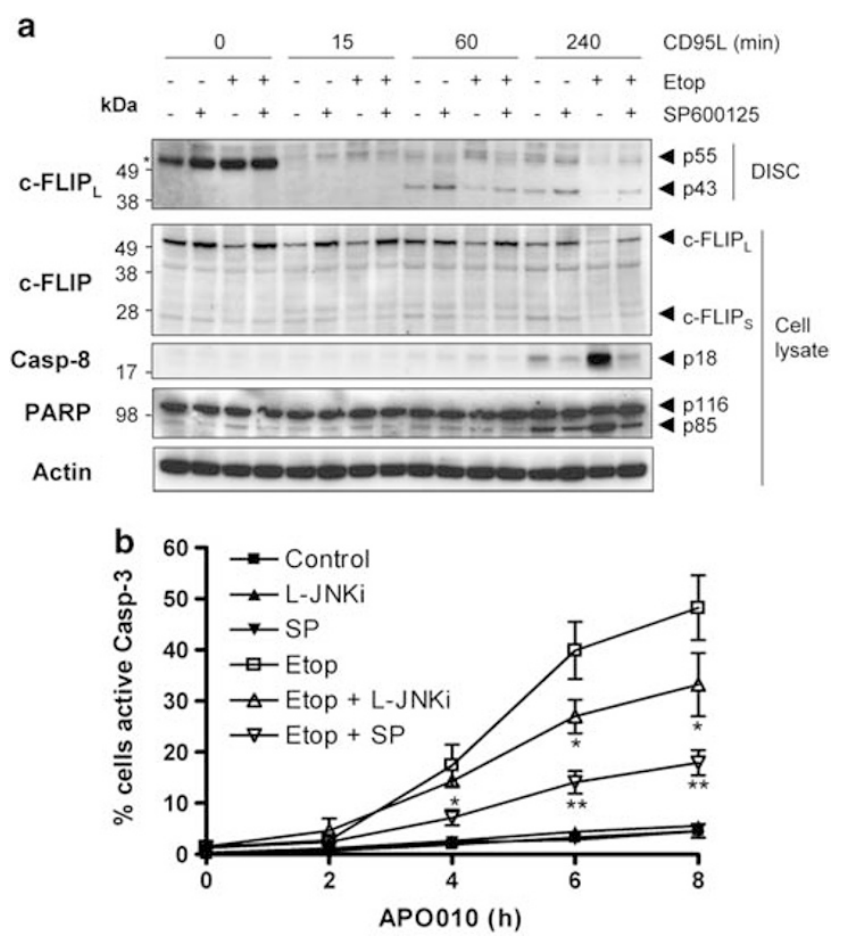

Figure 4 Inhibiting JNK signalling restores c-FLIP levels and overrules sensitization to APO010-induced apoptosis by etoposide. (a) J16-Bcl-2 cells were left untreated $(-)$ or incubated for 30 min with $10 \mu \mathrm{M} \mathrm{SP600125} \mathrm{(+).} \mathrm{Next,} \mathrm{cells}$ were incubated with $(+)$ or without $(-)$ etoposide (Etop) for $15 \mathrm{~h}$ and subsequently stimulated with $20 \mathrm{ng} / \mathrm{ml}$ FLAG-CD95L pre-coupled to biotinylated anti-FLAG antibody for the indicated periods of time. DISCs were isolated and C-FLIP protein levels in DISCs and total cell lysates were determined using immunoblotting. Caspase-8- (p18) and PARP (p85) processing were also determined in total cell lysates. Actin served as a loading control. (b) J16-Bcl-2 cells were incubated in the presence or absence of $10 \mu \mathrm{M} \mathrm{SP600125} \mathrm{(30} \mathrm{min)} \mathrm{or} 10 \mu \mathrm{M} \mathrm{L-JNKi} \mathrm{peptide}(24 \mathrm{~h})$ before treatment with $2 \mu \mathrm{g} / \mathrm{ml}$ etoposide. After $15 \mathrm{~h}$, cells were stimulated with $0.4 \mathrm{ng} / \mathrm{ml} \mathrm{APO010}$ and harvested for analysis of caspase-3 activation using flow cytometry at the indicated time points. The values represent mean \pm S.D. of three independent experiments. Statistically significant differences between the values of Etop and Etop + L-JNKi or Etop + SP samples are indicated as ${ }^{*} P<0.05$ and ${ }^{* *} P<0.01$

In summary, c-FLIP downregulation by RNAi greatly facilitated the mitochondrion-independent pathway for effector caspase activation and largely overruled the capability of etoposide to increment apoptotic execution. These data indicate that downregulation of C-FLIP protein expression is a major mechanism by which etoposide sensitizes J16-Bcl-2 cells to APO010-induced apoptosis.

Various stimuli that sensitize J16-Bcl-2 cells to APO010 downregulate c-FLIP. Next, we examined whether other stress stimuli could also facilitate APO010-induced apoptosis in $\mathrm{J} 16-\mathrm{Bcl}-2$ cells. For this purpose, cells were pre-treated with IR, UV radiation, the HDAC inhibitors, trichostatin $A$ (TSA) and valproic acid (VPA), or the proteasome inhibitors, MG132 and lactacystin. In all cases, pretreatment significantly enhanced APO010-induced apoptosis (Figures $6 a-d)$. In the time frame of pre-sensitization, IR slightly increased CD95 membrane levels (Figure 6f), but etoposide and UV had no effect, whereas VPA and TSA significantly 
a
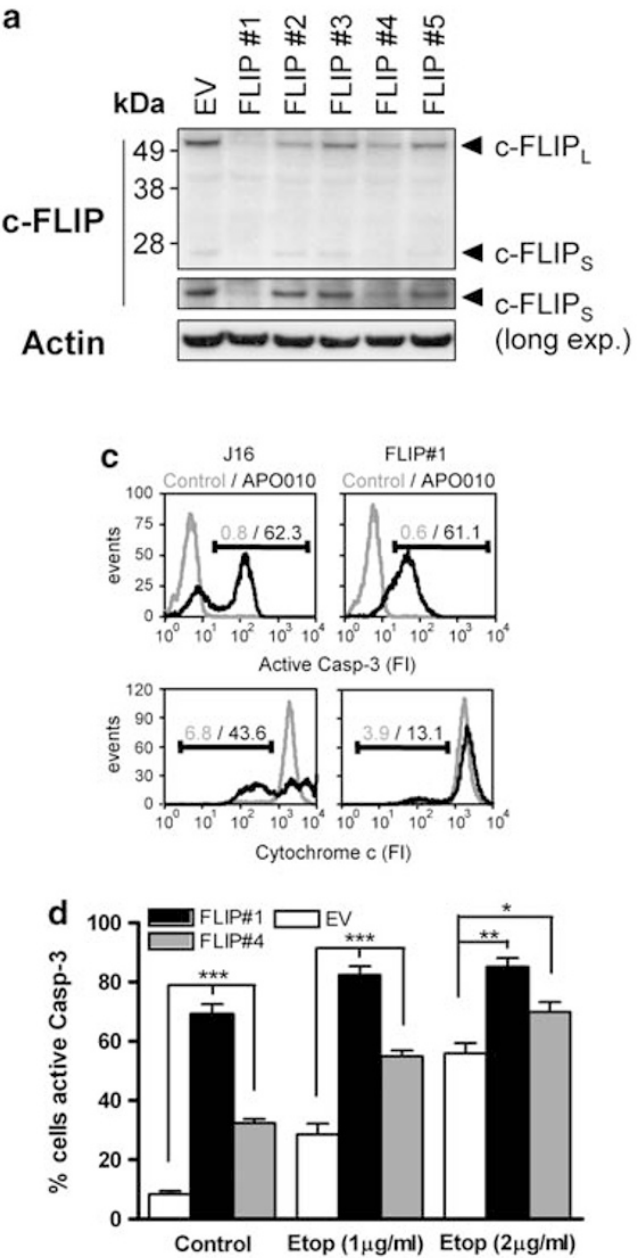

b
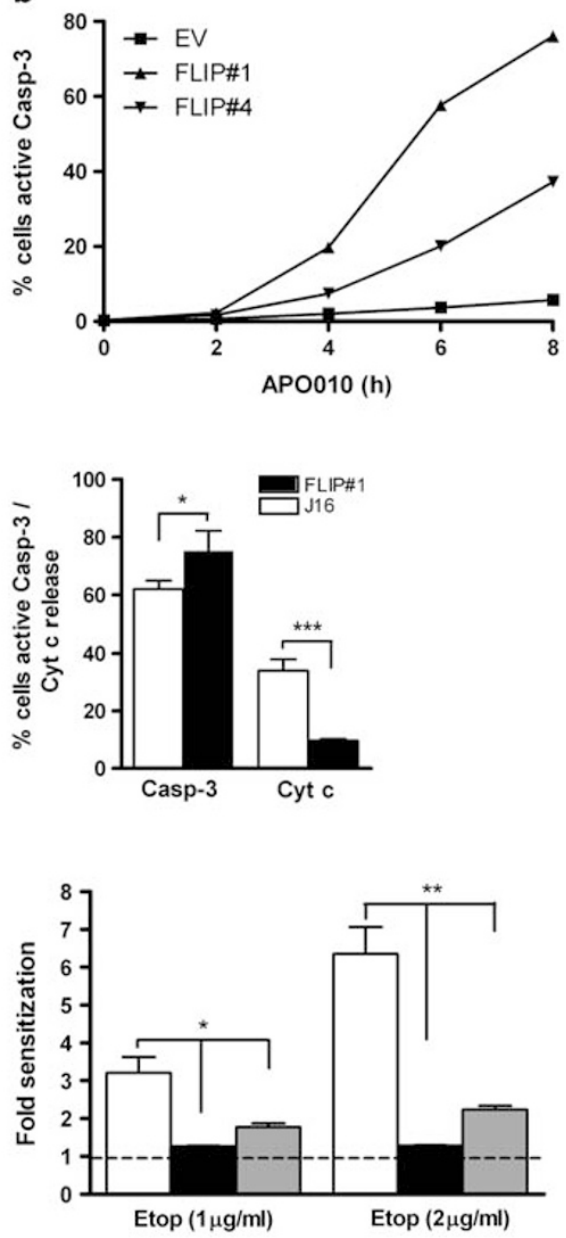

Figure 5 Downregulating c-FLIP levels facilitates the direct pathway for apoptotic execution by APO010 and significantly impairs sensitization of J16-Bcl-2 cells by etoposide. (a) C-FLIP protein levels determined using immunoblotting in total lysates of empty vector (EV)-transduced J16-Bcl-2 cells and in J16-Bcl-2 cells expressing RNAi constructs for c-FLIP (FLIP1-5). Actin served as a loading control. (b) EV, FLIP1 and FLIP4 J16-Bcl-2 cells were stimulated with $0.4 \mathrm{ng} / \mathrm{ml} \mathrm{APO010}$ and analyzed for active caspase-3 using flow cytometry at the indicated time points. (c) EV-transduced J16 cells (J16) or FLIP1 transduced J16-Bcl-2 cells (FLIP1) were stimulated with 0.4 ng/ml APO010 for $8 \mathrm{~h}$ or left unstimulated (control). Active caspase- 3 content and Cyt $c$ release were determined using flow cytometry. Flow cytometric plots in left panels are data from one representative experiment. The numbers in the panels represent percentage of events in the indicated gate. Fl, fluorescence intensity. The bar diagram (right panel) shows means + S.D. of three independent experiments. (d) EV-, FLIP1- or FLIP4-transduced J16-Bcl-2 cells were incubated for $15 \mathrm{~h}$ in the absence (Control) or presence of etoposide (Etop) at the indicated concentrations. Next, cells were stimulated with APO010 for $8 \mathrm{~h}$ and analyzed for active caspase-3 content. The values in left panel are mean + S.D. of 3-4 independent experiments. Background apoptosis at $t=0$, before stimulation with APO010 was subtracted (values: Control $<1 \%, 1 \mu \mathrm{g} / \mathrm{ml}$ etoposide $2.4-$ $3.1 \%, 2 \mu \mathrm{g} / \mathrm{ml}$ etoposide $2.7-5.8 \%$ ). Fold sensitization depicted in the right panel was extrapolated from caspase-3 activation data in the left panel and indicates the fold enhancement of APO010-induced caspase-3 activation by pre-treatment of J16-Bcl-2 cells with etoposide. Statistically significant differences between values of EV versus FLIP1 or FLIP4 samples are indicated as ${ }^{\star} P<0.05,{ }^{* \star} P<0.01$ and ${ }^{* \star *} P<0.001$

downregulated CD95. This ruled out CD95 upregulation at the plasma membrane as the mechanism for increased APO010 sensitivity.

In addition, we found no evidence that the modulation of inhibitor of apoptosis protein (IAP) expression was instrumental in the sensitization to APO010-induced apoptosis. First, treatment with etoposide or IR did not alter C-IAP-1, C-IAP-2 or XIAP mRNA or protein expression in J16 cells. ${ }^{16}$ Second, stable expression of a validated cytosolic SMAC mutant (J16-Bcl-2- $\Delta 55$ SMAC) ${ }^{16}$ did not abrogate the capacity of etoposide to sensitize J16-Bcl-2 cells to AP0010-induced apoptosis (Supplementary Figure 5).

The treatment of $\mathrm{J} 16-\mathrm{Bcl}-2$ cells with each of the stimuli did, however, downregulate total cellular c-FLIP protein levels
(Figure 6e). In the case of IR, UV and VPA, SP600125 could not overrule sensitization (Supplementary Figure 6), which correlated with an inability to restore c-FLIP levels (Supplementary Figure 7 and data not shown).

We conclude that in addition to etoposide, IR, UV radiation, HDAC and proteasome inhibitors also sensitized J16-Bcl-2 cells to APO010 apoptosis, which was correlated with c-FLIP downregulation.

Downregulation of c-FLIP by RNAi impairs the ability of different stimuli to sensitize to APO010. Subsequently, we used c-FLIP RNAi to test whether c-FLIP downregulation was a common mechanism by which the various stress stimuli sensitized J16-Bcl-2 cells to APO010-induced 

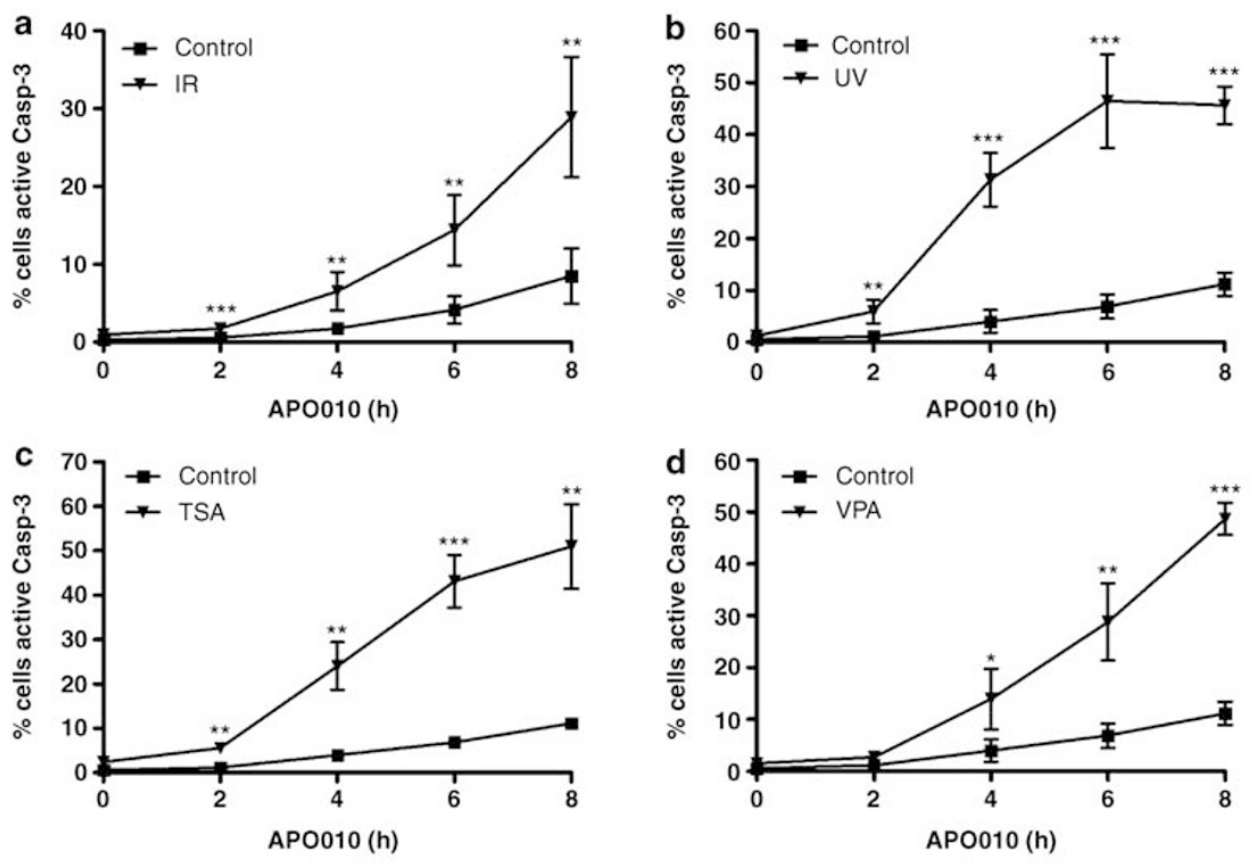

e

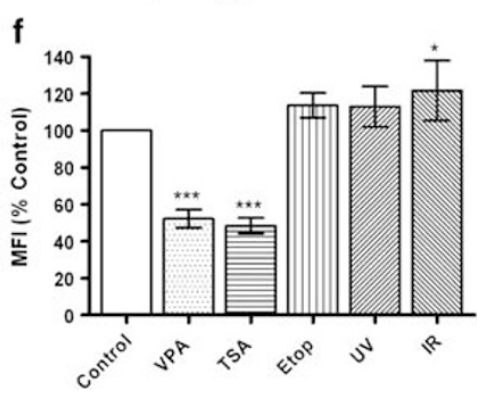

Figure 6 Various stimuli - which sensitize J16-Bcl-2 cells to APO010 - downregulate c-FLIP protein levels, without upregulating CD95 membrane levels. (a-d) J16-Bcl-2 cells were left untreated (Control) or stimulated with (a) $30 \mathrm{~Gy} \mathrm{IR,} \mathrm{(b)} 60 \mathrm{~J} / \mathrm{m}^{2} \mathrm{UV}$, (c) $300 \mathrm{nM}$ TSA or (d) $6.4 \mathrm{mM}$ VPA. Directly after stimulation (UV) or $15 \mathrm{~h} \mathrm{later} \mathrm{(IR,} \mathrm{TSA} \mathrm{and}$ VPA), cells were stimulated with $0.4 \mathrm{ng} / \mathrm{ml} \mathrm{APO010}$ for the indicated periods of time and analyzed for active caspase-3 content using flow cytometry. Data represent means \pm S.D. of at least three independent experiments. Statistically significant differences between values of Control and IR, UV, VPA or TSA treated cells are indicated as ${ }^{*} P<0.05,{ }^{* *} P<0.01$ and ${ }^{* \star *} P<0.001$. (e) J16-Bcl-2 cells were harvested at $15 \mathrm{~h}$ after stimulation with $2 \mu \mathrm{g} / \mathrm{ml}$ etoposide (Etop), 10 or $30 \mathrm{~Gy}$ IR, $300 \mathrm{nM}$ TSA, $10 \mu \mathrm{M}$ lactacystin (Lacta), $6.4 \mathrm{mM}$ VPA, $5 \mu \mathrm{M} \mathrm{MG132}$ or at $3 \mathrm{~h}$ after irradiation with $60 \mathrm{~J} / \mathrm{m}^{2} \mathrm{UV}$. Total c-FLIP protein levels were determined in cell lysates using immunoblotting. The open and filled triangles indicate the c-FLIPS and C-FLIPL isoforms, respectively. Actin served as a loading control. Brackets indicate samples derived from the same experiment, each with its own control. Data are representative of multiple experiments. (f) J16-Bcl-2 cells were stimulated with the indicated regimes as outlined in (e) and CD95 membrane levels of live cells were determined using flow cytometry. Values of mean fluorescence intensity (MFI) are represented as mean \pm S.D. of at least four independent experiments, normalized to CD95 MFI of untreated cells. Statistically significant differences between values of Control and VPA, TSA or IR treated samples are indicated as ${ }^{*} P<0.05$ and ${ }^{* *} P<0.001$

apoptosis. Control or FLIP4-transduced J16-Bcl-2 cells were pretreated with IR, UV, TSA, VPA, MG132 or lactacystin and next stimulated with APO010. After $8 \mathrm{~h}$, the cells were harvested and analyzed for active caspase-3 content using flow cytometry. All stimuli strongly sensitized control cells to APO010-induced caspase-3 activation (Figures $7 a$ and b). However, in FLIP4-transduced cells, which had intermediate c-FLIP levels (Figure 5a), sensitization was significantly reduced (Figure $7 \mathrm{~b}$ ).

These results indicate that C-FLIP downregulation is a common mechanism by which J16-Bcl-2 cells are sensitized to CD95-mediated apoptosis by a wide range of stimuli.

FLIP downregulation and sensitization to APO010induced apoptosis in a solid tumor cell line. To analyze the generality of this mechanism, we examined its occurrence in HCT-15 colon carcinoma cells, which are p53 mutant ${ }^{23}$ and have been documented as type II cells. ${ }^{24}$ HCT -15 cells were retrovirally transduced with Bcl-2 to block the mitochondrial pathway for caspase activation (Supplementary Figure $8 \mathrm{~b}$ ). The cells were not pretreated (Control) and subsequently stimulated with APO010, or pretreated with etoposide, IR, UV radiation or HDAC inhibitor VPA and subsequently stimulated with APO010. These sensitizing stimuli alone did not induce caspase-3 activation (or apoptosis) in HCT-15-Bcl-2 cells (Supplementary Figure 8a; O h APO010). In addition, in agreement with the documented type II nature of HCT-15 cells, APO010 alone induced only a very low level of caspase-3 activation in HCT-15-Bcl-2 cells. Sensitization of HCT-15-Bcl-2 cells to 

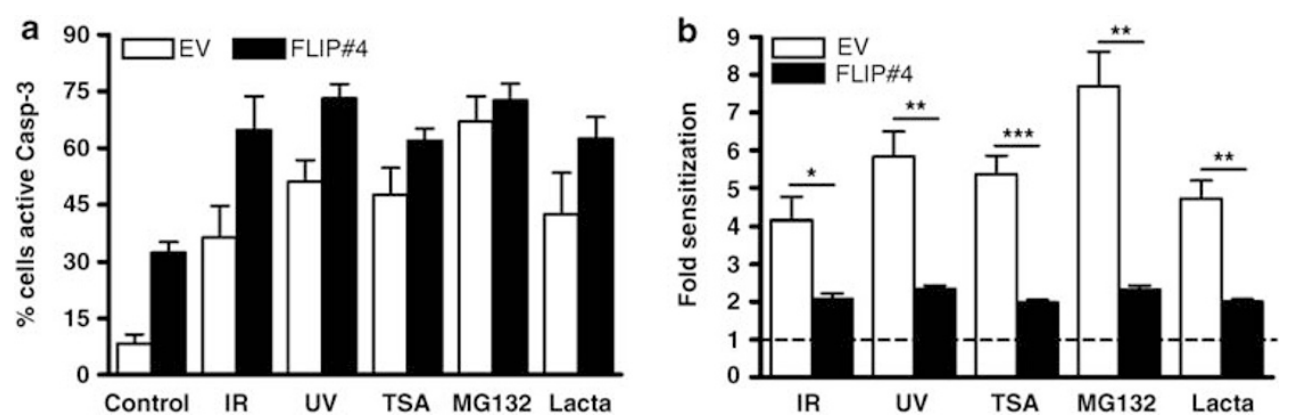

Figure 7 c-FLIP downregulation by RNAi impairs sensitization to APO010 by various stimuli. Empty vector (EV)- and FLIP4-transduced J16-Bcl-2 cells were stimulated with $60 \mathrm{~J} / \mathrm{m}^{2} \mathrm{UV}$, or incubated for $15 \mathrm{~h}$ after stimulation with $30 \mathrm{~Gy}$ IR, $300 \mathrm{nM} \mathrm{TSA}, 5 \mu \mathrm{M}$ MG132 or $10 \mu \mathrm{M}$ lactacystin (Lacta). Next, cells were stimulated with $0.4 \mathrm{ng} / \mathrm{ml}$ APO010 and analyzed $8 \mathrm{~h}$ later for active caspase-3 content using flow cytometry. (a) Values of caspase-3 activation represented as means + S.D. of 3-4 independent experiments. Background apoptosis at $t=0$ before stimulation with APO010 was subtracted (values: Control $<1 \%$, IR 2.2-5.1\%, TSA 3.5-5\%, MG132 15.6-19.1\%, lactacystin 9.3-13\%, UV 3.3-4.9\%). (b) Fold sensitization as extrapolated from caspase-3 activation data in (a). It indicates the fold enhancement of APO010-induced caspase-3 activation by pre-treatment of $\mathrm{J} 16-\mathrm{Bcl}-2$ cells with the indicated stimuli. Statistically significant differences between the indicated values are indicated as ${ }^{*} P<0.05$, ${ }^{* \star} P<0.01$ and ${ }^{* \star *} P<0.001$

APO010-induced apoptosis by UV radiation and VPA was very strong $(P<0.0001$ at $t=8 \mathrm{~h}$; Supplementary Figure $8 \mathrm{a})$ and these stimuli concomitantly downregulated c-FLIP protein levels (Supplementary Figure 8c). IR yielded a very low level of sensitization of HCT-15-Bcl-2 cells to APO010induced apoptosis $(P=0.015$ at $t=8 \mathrm{~h})$ and did not measurably downregulate c-FLIP protein levels. Therefore, in these cases, a correlation between sensitization to APO010 and C-FLIP downregulation was apparent. Etoposide, however, sensitized HCT-15-Bcl-2 cells to APO010-induced apoptosis at intermediate levels $(P=0.001$ at $t=8 \mathrm{~h}$; Supplementary Figure $8 \mathrm{a})$, yet did not detectably downregulate C-FLIP protein levels (Supplementary Figure 8c). Sensitization to any of these stimuli was not correlated with downregulation of XIAP, ClAP1 or clAP2 protein levels (Supplementary Figure $8 d$ ).

These results indicate that C-FLIP downregulation may contribute to, but is not the only mechanism, by which type II tumor cells can be sensitized to APO010-induced apoptosis.

\section{Discussion}

Many reports describe the combined effects of conventional or targeted anticancer regimens and death receptor agonists in tumor cell killing. For TRAIL receptor agonists in particular, combination treatment proved effective in a variety of tumor types in vitro and in vivo. ${ }^{6}$ The mechanisms of the action of death receptors and conventional anticancer regimens predict additivity in combined treatment. Moreover, upregulation of death receptors at the plasma membrane in response to DNAdamaging regimens may lead to synergy, in particular in p53proficient cells. ${ }^{6}$ However, in various cell lines, such as Jurkat leukemia, ${ }^{16} \mathrm{HT}-29$ colon carcinoma ${ }^{25}$ and Hep3b hepatocellular carcinoma, ${ }^{26}$ the combined effects of TRAIL and DNAdamaging regimens could not be explained by changes in receptor membrane expression, raising the question of the underlying molecular mechanism.

In this study we show that pretreatment with not only conventional anticancer regimens, such as IR and etoposide, but also UV radiation, HDAC inhibitors and proteasome inhibitors sensitized J16-Bcl-2 cells to APO010-induced apoptosis, in the absence of CD95 upregulation and without breaking mitochondrial resistance. In the time frame in which caspase-3 activation was observed - as read out by occurrence of its cleaved form and cleavage of its substrate PARP - Cyt $c$ release did not take place. In agreement with this, we previously found that downregulation of Bak and Bax by RNAi in J16-Bcl-2 cells did not alter the combined response to IR and TRAIL. ${ }^{16}$ Sensitization resulted in improved caspase- 8 activation by CD95 and execution through the direct pathway for effector caspase activation, which could be explained by C-FLIP downregulation.

Most likely, the reduction in c-FLIP protein levels induced by the different stimuli examined in this study allowed fewer c-FLIP molecules to be recruited into the CD95L DISC, thereby altering the C-FLIP/caspase-8/10 ratio. C-FLIP has a dual role in the DISC, as it inhibits caspase-8/10 activation when present at high levels, but can promote caspase-8/10 activation when present at low levels. ${ }^{10}$ We found that c-FLIP downregulation facilitated caspase-8/10 activation in the CD95L DISC. This agrees with other studies: in SHEP neuroblastoma cells, cycloheximide or actinomycin $D$ treatment reduced cellular c-FLIP protein levels and participation in the CD95L-DISC and incremented inducer caspase activation. ${ }^{27}$ Prostate carcinoma ${ }^{28}$ and Hodgkin/Reed-Sternberg cells ${ }^{29}$ were sensitized to CD95-mediated apoptosis by c-FLIP RNAi. Moreover, melanoma cells were sensitized to both CD95-and TRAIL-receptor-induced apoptosis by c-FLIP $\mathrm{RNAi}^{30}$ On the basis of current data, downregulation of c-FLIP - which is overexpressed in several tumor types - was recently proposed as a rational strategy to sensitize tumor cells to death ligand therapy. ${ }^{31}$ Future studies should pinpoint whether downregulation of the long or short isoform of c-FLIP is the critical determinant in sensitizing Jurkat-Bcl-2 cells to APO010-induced apoptosis. Both isoforms can be recruited to the DISC. ${ }^{32}$ Downregulation of $C-F L I P_{L}$ was most consistently observed, but c-FLIP levels were also affected. To resolve this issue, an isoform-specific RNAi approach is required. 
We previously found that pre-treatment of $\mathrm{J} 16-\mathrm{Bcl}-2$ cells with IR improved effector caspase-8/10 activation in the DISC and thereby allowed cellular execution by TRAIL through the direct pathway for effector caspase activation. ${ }^{16}$ In this study we show that in the same cells, c-FLIP downregulation by various stress stimuli allowed APO010 to bypass the mitochondrial pathway for apoptotic execution. These findings imply that the stress stimuli promote a 'switch' from type II to type I death receptor signalling in J16-Bcl-2 cells. In addition, in type II CEM leukemia cells, cycloheximide pretreatment allowed CD95-mediated apoptosis through the direct pathway. ${ }^{33}$ It is important to determine the generality of the mechanism defined in this study for J16-Bcl-2 cells and, in particular, whether it operates in solid tumors also. We have examined the case of HCT-15 colon carcinoma cells, as these have been well defined in terms of p53 mutant and type II status. UV and VPA could sensitize these cells to APO010-induced apoptosis, but IR was less effective. With these stimuli, a correlation between c-FLIP downregulation and sensitization to APO010-induced apoptosis was found. However, the correlation was not evident in the case of etoposide, which sensitized HCT-15-Bcl-2 cells to APO010 in the absence of c-FLIP downregulation. In SHEP neuroblastoma cells overexpressing Bcl-2, ${ }^{27}$ we found a correlation between FLIP downregulation and sensitization by UV, but not with the other stimuli (data not shown). Therefore, cells may be sensitized by other mechanisms to undergo an apparent type II to type I switch. In addition, in other type II cells (mesothelioma and carcinoma cell lines), an intact mitochondrial amplification loop was required for apoptotic execution upon combined treatment with DNA-damaging regimens and TRAIL, even though inducer caspase activity was enhanced. ${ }^{34-36}$ Therefore, enhanced inducer caspase activation may be sufficient in some cell types for execution through the direct pathway, whereas in other cell types - perhaps particularly in solid tumors - the mitochondrial pathway may still be required.

Concerning sensitization to TRAIL-induced apoptosis, a number of studies report a correlation between c-FLIP downregulation by cellular stressors and increased sensitivity to apoptosis. ${ }^{31}$ In colon ${ }^{25}$ and hepatocellular ${ }^{26}$ carcinoma, C-FLIP downregulation by 5-FU was correlated with reduced levels of $\mathrm{c}$-FLIP in the TRAIL DISC and with enhanced sensitivity to TRAIL-mediated apoptosis. However, we found that $10 \mathrm{~Gy}$ IR did not detectably alter c-FLIP levels in J16-Bcl-2 cells and did not sensitize them to APO010induced apoptosis (data not shown), whereas it did improve TRAIL DISC activity and apoptosis induction. ${ }^{16}$ This suggests that CD95 and TRAIL receptors have partially distinct requirements to activate inducer caspases in the DISC.

In J16-Bcl-2 cells, IR, UV radiation, etoposide, HDAC and proteasome inhibitors all downregulated c-FLIP protein levels. c-FLIP expression is tightly controlled, both at the transcriptional and at the protein level. The $\mathrm{NF}-\kappa \mathrm{B}$ pathway can induce c-FLIP transcription, although the ubiquitin-proteasome pathway mediates c-FLIP degradation. ${ }^{37,38} \mathrm{c}$-FLIP molecules have a short half-life and are subject to proteasomal degradation at the steady state. In addition, they are ubiquitinated in response to death receptor stimulation, through a defined ubiquitin ligase that is under the control of the JNK pathway. ${ }^{19,38}$ In agreement with this, short-term incubation with proteasome inhibitor rescued the disappearance of c-FLIP after addition of the stress stimuli, but upon prolonged incubation, c-FLIP was still downregulated (results not shown). This can be explained by the fact that proteasome activity is required for NF- $\kappa \mathrm{B}$ activation, which in turn directs c-FLIP transcription.

In case of etoposide, we found that JNK inhibition impeded c-FLIP downregulation. This concurs with the report that implicated JNK in c-FLIP ubiquitination ${ }^{19}$ and with the finding that JNK inhibition reversed etoposide-mediated sensitization to TRAIL in mesothelioma cells. ${ }^{20}$ In case of UV radiation, however, which potently activated the JNK pathway and downregulated c-FLIP, the JNK inhibitors - although functional - could not rescue c-FLIP protein expression. Only in case of IR, c-FLIP levels were partially restored upon JNK inhibition (Supplementary Figure 7). The extent of c-FLIP restoration correlated with the extent to which sensitization to APO010 was reversed (Supplementary Figure 6). Therefore, JNK activation may contribute to, but is not a general mechanism by which c-FLIP is downregulated in response to stress stimuli, and JNK activation per se does not sensitize cells for APO010-induced apoptosis.

In conclusion, c-FLIP downregulation is a common mechanism by which various stress inputs sensitize J16-Bcl-2 cells to CD95-mediated apoptosis through the direct pathway for caspase activation. The apparent ability of death receptors to 'switch' from type II to type I signalling after c-FLIP downregulation may be of more general relevance for sensitizing tumor cells to death receptor-mediated apoptosis. It will be of interest to determine in which primary tumor types this is the case.

\section{Materials and Methods}

Cell lines and reagents. The J16 Jurkat clone stably overexpressing Bcl-2 and luciferase (J16-Bcl-2) was generated by retroviral transduction as described. ${ }^{39}$ $J 16$ cells are heterozygous mutant for $p 53$ and the DNA damage-induced apoptosis pathway is p53 independent. ${ }^{39} \mathrm{~J} 16-\mathrm{Bcl}-2$ cells and derivatives were cultured in Iscove's modified Dulbecco's medium with $8 \%$ fetal bovine serum and antibiotics. AP0010 in native and biotinylated form was kindly provided by TopoTarget (Lausanne, Switzerland), produced as described ${ }^{14}$ and kept frozen in storage buffer at $-80^{\circ} \mathrm{C}$. SP600125 was from ENZO Life Sciences International Inc. (Zandhoven, Belgium).The cell permeable L-JNKi peptide (sequence NGRKKRRQRRRPPR PKRPTTLNLFPQVPRSQD with carboxy-terminal amide) was produced at the Division of Cell Biology at the Netherlands Cancer Institute, according to the standard procedures. The product was purified by HPLC and its sequence was verified using mass spectrometry. TSA, VPA, anti-caspase-8 monoclonal antibody (mAb, clone C15), anti-FLIP mAb (clone NF6) and soluble human FLAG-tagged CD95L were obtained from Alexis Biochemicals (Lausanne, Switzerland). Biotinylated anti-FLAG $\mathrm{mAb}$ (clone M2), etoposide, puromycin and blasticidin $\mathrm{S}$ were from Sigma-Aldrich (St. Louis, MO, USA). Anti-Cyt c mAb (clone 6H2.B4), anti-XIAP mAb and rabbit antiactive caspase-3 antibody were from BD Biosciences (San Jose, CA, USA), anti-actin $\mathrm{mAb}$ (clone C4) was from Chemicon International (Temecula, CA, USA) and rabbit anti-clAP1 and anti-clAP2 were from R\&D Systems (Abingdon, UK). AlexaFluor 647conjugated goat anti-mouse $\mathrm{Ig}$ and goat anti-rabbit Ig were from Molecular Probes (Leiden, The Netherlands). Lactacystin and MG132 were from Calbiochem (Darmstadt, Germany). Horseradish peroxidase-conjugated rabbit anti-mouse Ig and swine anti-rabbit Ig were from DAKO A/S (Glostrup, Denmark) and the enhanced chemiluminescence kit was from Pierce Biotechnology (Rockford, IL, USA). Rabbit anti-PARP polyclonal Ab (pAb) 9542 was from Cell Signaling Technologies (Danvers, MA, USA). Streptavidin-conjugated sepharose beads were from Zymed (San Francisco, CA, USA). 
Retroviral gene transduction. Stable knockdown of c-FLIP in J16-Bcl-2 cells with si RNA (complementary sense and antisense oligonucleotides FLIP1: $5^{\prime}$ GAATAGACCTGAAGACAAA-3', FLIP2: 5'-GATACAAGATGAAGAGCAA-3', FLIP3: 5'-GCTATGAAGTCCAGAAATT-3', FLIP4: 5'-GGAGCAGGGACAAGTTACA-3', FLIP5: $5^{\prime}$-GCAAGGAGAAGAGTTTCTT-3') was performed with the pRSC retroviral vector, which is a modification of pRetroSuper ${ }^{40}$ with a puromycin resistance cassette. Packaging of the (dideoxynucleotide sequence verified) constructs in the HT1080 packaging cell line FLY and transduction of J16-Bcl-2 cells with freshly harvested virus supernatant was carried out as described. ${ }^{16}$ Cells were selected after 3 days with $1 \mu \mathrm{g} / \mathrm{ml}$ puromycin.

Apoptosis assays. For apoptosis assays, cells were stimulated with the indicated doses of APO010, etoposide, IR, TSA, VPA, MG132 or lactacystin in their normal medium. After addition of stimulus, cells were incubated for the indicated time periods at $37{ }^{\circ} \mathrm{C}$ in $5 \% \mathrm{CO}_{2}$. IR was delivered using a ${ }^{137} \mathrm{Cs}$ source $(415 \mathrm{Ci}$; Von Gahlen Nederland, B.V., Zevenaar, The Netherlands) at an absorbed dose rate of approximately $0.6 \mathrm{~Gy} / \mathrm{min}$. For UV irradiation, $5 \times 10^{5}$ cells were resuspended in $500 \mu \mathrm{l}$ culture medium and transferred into 12-well plate. After allowing cells to sink to the bottom of the plate for at least $30 \mathrm{~min}$, UV irradiation was performed using the UV Stratalinker 2400 (Stratagene, La Jolla, CA, USA) at a dose rate of $10 \mathrm{~J} / \mathrm{m}^{2} / \mathrm{s}$. In some experiments, cells were incubated (before sensitization) with $10 \mu \mathrm{M}$ SP600125 for $30 \mathrm{~min}$ or $10 \mu \mathrm{M} \mathrm{L-JNKi} \mathrm{peptide} \mathrm{for} 24 \mathrm{~h}$ at $37^{\circ} \mathrm{C}$ in $5 \% \mathrm{CO}_{2}$. Nuclear fragmentation was determined using flow cytometric analysis of PI-stained nuclei as described. ${ }^{16}$ Data were analyzed using FCS Express (De Novo Software, Thornhill, Canada). Cellular debris was excluded from analyses and the percentage of subdiploid PI-positive particles was scored as percentage apoptosis. Active caspase- 3 content and Cyt $c$ release using flow cytometry were determined as described. ${ }^{16}$

DISC isolation. J16-Bcl-2 cells were stimulated with $2 \mu \mathrm{g} / \mathrm{ml}$ etoposide and $15 \mathrm{~h}$ later seeded at $10^{8} \mathrm{cells} / \mathrm{sample}$ in $30 \mathrm{ml}$ medium in a $15-\mathrm{cm}$ dish. Cells were stimulated with $20 \mathrm{ng} / \mathrm{ml}$ FLAG-CD95L pre-coupled to $100 \mathrm{ng} / \mathrm{ml}$ biotinylated antiFLAG M2 mAb for the indicated periods of time. DISC isolation was performed as described ${ }^{16}$ with some modifications of the original protocol. ${ }^{9}$

Immunoblotting. Total cellular protein was determined using Bio-Rad protein assay (München, Germany) and was separated at $40 \mu \mathrm{g}$ per lane on $4-12 \%$ NuPage Bis-Tris gradient gels (Invitrogen, Breda, The Netherlands) in MES buffer, according to the manufacturer's instructions. Subsequent immunoblotting was performed as described in Verbrugge et $a{ }^{16}{ }^{16}$ and the references in that study. For some blots, the iBlot system (Invitrogen) was used according to the manufacturer's instructions. Where appropriate, autoradiography signals were quantified using a Fluorchem8000 chemiluminescence imager (Alpha Innotech Corp., San Leandro, CA, USA).

Flow cytometric detection of CD95. For flow cytometric detection of CD95 surface levels, cells were incubated for $15 \mathrm{~h}$ with the indicated stimuli at $37^{\circ} \mathrm{C}$ in $5 \% \mathrm{CO}_{2}$. After incubation, cells were stained with biotinylated APO010 and subsequently with streptavidin-conjugated allophycocyanine (BD Pharmingen, San Diego, CA, USA). Samples were gated on live, Pl-negative cells and data were analyzed using FCS Express. Mean fluorescence intensity values of stimulated cells were normalized to those of unstimulated (Control) cells.

Statistics. The differences between treatment groups were analyzed using unpaired Student's $t$-tests and were considered significant when $P<0.05$. The analyses were performed using GraphPad Prism version 4 for Windows (Graph Pad Software, San Diego, CA, USA).

Acknowledgements. We thank our collaborators at TopoTarget Switzerland SA and Apoxis SA (Lausanne, Switzerland), in particular Nicola Beltraminelli, for making APO010 available to us, Henk Hilkmann for production of the L-JNKi peptide and Ernst-Jan Geutjes for help with qRT-PCR. This work was financially supported by the Dutch Cancer Society (project NKI 2004-3079).

1. Hanahan D, Weinberg RA. The hallmarks of cancer. Cell 2000; 100: 57-70.

2. Brown JM, Wouters BG. Apoptosis, p53, and tumor cell sensitivity to anticancer agents. Cancer Res 1999; 59: 1391-1399.
3. Strasser A, Harris AW, Jacks T, Cory S. DNA damage can induce apoptosis in proliferating lymphoid cells via p53-independent mechanisms inhibitable by Bcl-2. Cell 1994; 79: 329-339.

4. Wang X. The expanding role of mitochondria in apoptosis. Genes Dev 2001; 15: 2922-2933.

5. Schmitt CA, Rosenthal CT, Lowe SW. Genetic analysis of chemoresistance in primary murine lymphomas. Nat Med 2000; 6: 1029-1035.

6. Ashkenazi A, Herbst RS. To kill a tumor cell: the potential of proapoptotic receptor agonists J Clin Invest 2008; 118: 1979-1990.

7. Ashkenazi A, Holland P, Eckhardt G. Ligand-based targeting of apoptosis in cancer: the potential of recombinant human apoptosis ligand 2/tumor necrosis factor-related apoptosis-inducing ligand (rhApo2L/TRAIL). J Clin Oncol 2008; 26: 3621-3630.

8. Peter ME, Krammer PH. The CD95(APO-1/Fas) DISC and beyond. Cell Death Differ 2003; 10: 26-35.

9. Sprick MR, Rieser E, Stahl H, Grosse-Wilde A, Weigand MA, Walczak H. Caspase-10 is recruited to and activated at the native TRAIL and CD95 death-inducing signalling complexes in a FADD-dependent manner but can not functionally substitute caspase-8. EMBO J 2002; 21: 4520-4530.

10. Chang DW, Xing Z, Pan Y, Algeciras-Schimnich A, Barnhart BC, Yaish-Ohad S et al. $c-F L I P(L)$ is a dual function regulator for caspase-8 activation and CD95-mediated apoptosis. EMBO J 2002; 21: 3704-3714.

11. Barnhart BC, Alappat EC, Peter ME. The CD95 type I/type II model. Semin Immunol 2003; 15: 185-193.

12. Ogasawara J, Watanabe-Fukunaga R, Adachi M, Matsuzawa A, Kasugai T, Kitamura $Y$ et al. Lethal effect of the anti-Fas antibody in mice. Nature 1993; 364: 806-809.

13. Holler N, Tardivel A, Kovacsovics-Bankowski M, Hertig S, Gaide O, Martinon F et al. Two adjacent trimeric Fas ligands are required for Fas signaling and formation of a deathinducing signaling complex. Mol Cell Biol 2003; 23: 1428-1440.

14. Verbrugge I, Wissink EH, Rooswinkel RW, Jongsma J, Beltraminelli N, Dupuis M et al. Combining radiotherapy with APO010 (MegaFas Ligand) in cancer treatment, a preclinical evaluation of feasibility. Clin Cancer Res 2009; 15: 2031-2038.

15. Scaffidi C, Fulda S, Srinivasan A, Friesen C, Li F, Tomaselli KJ et al. Two CD95 (APO-1/ Fas) signaling pathways. EMBO J 1998; 17: 1675-1687.

16. Verbrugge I, de Vries E, Tait SW, Wissink EH, Walczak H, Verheij M et al. Ionizing radiation modulates the TRAIL death-inducing signaling complex, allowing bypass of the mitochondrial apoptosis pathway. Oncogene 2008; 27: 574-584.

17. Huang DC, Hahne M, Schroeter M, Frei K, Fontana A, Villunger A et al. Activation of Fas by FasL induces apoptosis by a mechanism that cannot be blocked by $\mathrm{Bcl}-2$ or $\mathrm{Bcl}-\mathrm{X}(\mathrm{L})$. Proc Natl Acad Sci USA 1999; 96: 14871-14876.

18. Rudner J, Lepple-Wienhues A, Budach W, Berschauer J, Friedrich B, Wesselborg S et al. Wild-type, mitochondrial and ER-restricted Bcl-2 inhibit DNA-damage-induced apoptosis, but do not affect death receptor-induced apoptosis. J Cell Sci 2001; 114: 4161-4172.

19. Chang L, Kamata H, Solinas G, Luo JL, Maeda S, Venuprasad K et al. The E3 ubiquitin ligase itch couples JNK activation to TNFalpha-induced cell death by inducing $c-F L I P(L)$ turnover. Cell 2006; 124: 601-613.

20. Vivo C, Liu W, Broaddus VC. c-Jun N-terminal kinase contributes to apoptotic synergy induced by tumor necrosis factor-related apoptosis-inducing ligand plus DNA damage in chemoresistant, p53 inactive mesothelioma cells. J Biol Chem 2003; 278: 25461-25467.

21. Bain J, Plater L, Elliott M, Shpiro N, Hastie CJ, McLauchlan H et al. The selectivity of protein kinase inhibitors: a further update. Biochem J 2007; 408: 297-315.

22. Bonny C, Oberson A, Negri S, Sauser C, Schorderet DF. Cell-permeable peptide inhibitors of JNK: novel blockers of beta-cell death. Diabetes 2001; 50: 77-82.

23. Mashima T, Oh-hara T, Sato S, Mochizuki M, Sugimoto Y, Yamazaki K et al. p53-defective tumors with a functional apoptosome-mediated pathway: a new therapeutic target. J Natl Cancer Inst 2005; 97: 765-777.

24. Algeciras-Schimnich A, Pietras EM, Barnhart BC, Legembre P, Vijayan S, Holbeck SL et al. Two CD95 tumor classes with different sensitivities to antitumor drugs. Proc Natl Acad Sci USA 2003; 100: 11445-11450.

25. Lacour S, Micheau O, Hammann A, Drouineaud V, Tschopp J, Solary E et al. Chemotherapy enhances TNF-related apoptosis-inducing ligand DISC assembly in HT29 human colon cancer cells. Oncogene 2003; 22: 1807-1816.

26. Ganten TM, Haas TL, Sykora J, Stahl H, Sprick MR, Fas SC et al. Enhanced caspase-8 recruitment to and activation at the DISC is critical for sensitisation of human hepatocellular carcinoma cells to TRAIL-induced apoptosis by chemotherapeutic drugs. Cell Death Differ 2004; 11: S86-S96.

27. Fulda S, Meyer E, Debatin KM. Metabolic inhibitors sensitize for CD95 (APO-1/Fas)induced apoptosis by down-regulating Fas-associated death domain-like interleukin 1-converting enzyme inhibitory protein expression. Cancer Res 2000; 60: 3947-3956.

28. Hyer ML, Sudarshan S, Kim Y, Reed JC, Dong JY, Schwartz DA et al. Downregulation of c-FLIP sensitizes DU145 prostate cancer cells to Fas-mediated apoptosis. Cancer Biol Ther 2002; 1: 401-406.

29. Mathas S, Lietz A, Anagnostopoulos I, Hummel F, Wiesner B, Janz M et al. c-FLIP mediates resistance of Hodgkin/Reed-Sternberg cells to death receptor-induced apoptosis. J Exp Med 2004; 199: 1041-1052.

30. Geserick $P$, Drewniok $C$, Hupe $M$, Haas TL, Diessenbacher $P$, Sprick MR et al. Suppression of CFLIP is sufficient to sensitize human melanoma cells to TRAIL- and CD95L-mediated apoptosis. Oncogene 2008; 27: 3211-3220. 
31. Safa AR, Day TW, Wu CH. Cellular FLICE-like inhibitory protein (C-FLIP): a novel target for cancer therapy. Curr Cancer Drug Targets 2008; 8: 37-46.

32. Golks A, Brenner D, Fritsch C, Krammer PH, Lavrik IN. c-FLIPR, a new regulator of death receptor-induced apoptosis. J Biol Chem 2005; 280: 14507-14513.

33. Brumatti G, Yon M, Castro FA, Bueno-da-Silva AE, Jacysyn JF, Brunner $T$ et al. Conversion of CD95 (Fas) type II into type I signaling by sub-lethal doses of cycloheximide. Exp Cell Res 2008; 314: 554-563.

34. Broaddus VC, Dansen TB, Abayasiriwardana KS, Wilson SM, Finch AJ, Swigart LB et al. Bid mediates apoptotic synergy between tumor necrosis factor-related apoptosis-inducing ligand (TRAIL) and DNA damage. J Biol Chem 2005; 280: 12486-12493.

35. Ravi R, Bedi A. Requirement of BAX for TRAIL/Apo2L-induced apoptosis of colorectal cancers: synergism with sulindac-mediated inhibition of Bcl-x(L). Cancer Res 2002; 62 : 1583-1587.
36. Ramp U, Caliskan E, Mahotka C, Krieg A, Heikaus S, Gabbert HE et al. Apoptosis induction in renal cell carcinoma by TRAIL and gamma-radiation is impaired by deficient caspase-9 cleavage. Br J Cancer 2003; 88: 1800-1807.

37. Yu JW, Shi Y. FLIP and the death effector domain family. Oncogene 2008; 27: 6216-6227.

38. Chanvorachote P, Nimmannit U, Wang L, Stehlik C, Lu B, Azad N et al. Nitric oxide negatively regulates Fas CD95-induced apoptosis through inhibition of ubiquitinproteasome-mediated degradation of FLICE inhibitory protein. J Biol Chem 2005; 280: 42044-42050.

39. Wissink EH, Verbrugge I, Vink SR, Schader MB, Schaefer U, Walczak H et al. TRAIL enhances efficacy of radiotherapy in a p53 mutant, Bcl-2 overexpressing lymphoid malignancy. Radiother Oncol 2006; 80: 214-222.

40. Brummelkamp TR, Bernards R, Agami R. Stable suppression of tumorigenicity by virusmediated RNA interference. Cancer Cell 2002; 2: 243-247.

\section{Supplementary Information accompanies the paper on Cell Death and Differentiation website (http://www.nature.com/cdd)}

